\title{
Assessment of Error Estimators for Grid Adaptation in Large Eddy Simulation
}

\author{
Yao Jiang \\ Department of Mechanical Engineering \\ McGill University \\ Montreal, Canada \\ yao.jiang@mail.mcgill.ca
}

\author{
Siva Nadarajah \\ Department of Mechanical Engineering \\ McGill University \\ Montreal, Canada \\ siva.nadarajah@mcgill.ca
}

\begin{abstract}
Mesh adaptation relies on an error estimation process. Error estimators should properly take into consideration the contribution from both numerical and modeling related errors. Numerical dissipation plays an important contribution to the effective modeled turbulent kinetic energy (TKE), and must be accounted for. In the present paper, a novel approach is proposed for the estimation of numerical TKE. Several error estimators for LES are implemented and assessed for grid adaptation. The validation of the periodic hill test case was carried out for the coarse, the fine and the adapted grids. Numerical results are validated through a comparison against reference LES and experimental data. We employ and demonstrate the use of various error estimators for mesh adaptation.
\end{abstract}

Index Terms - mesh adaptation, large eddy simulation, error estimator

\section{INTRODUCTION}

The application of Large Eddy Simulation (LES) usually demands exorbitant computational cost. Mesh adaptation provides an ability to efficiently and strategically use computational resources. The process is typically based on a quality assessment process or an error estimator. LES intends to resolve the large scales while models the small via a SubgridScale (SGS) model. From an error analysis point of view, both the numerical and modeling errors contribute towards the total error [1], [2]. The distinction of the two sources of error is artificial and in practice the two interact and act as a whole and both are affected by the grid size.

Existing error estimators and mesh adaptation procedures for Reynolds-averaged Navier-Stokes (RANS) solvers could serve as references [3] but cannot be directly applied to LES due to the nature of the turbulence model. Despite the number of literature devoted to the assessment of LES quality [4]-[9], few of them could be directly applied to mesh adaptation for practical LES applications, due to the following limitations:

1) Different from RANS, where the grid size mainly affects the discretization error; in LES, both modeling and numerical errors are implicitly dependent on each other and are non-trivial to estimate;

2) There is no grid-convergence for LES since as the grid is refined, smaller scales are developed in the solution;

3) LES is intrinsically unsteady and chaotic, such that the error estimators should include some averaging process in space and/or time;
4) Different from detailed LES quality assessment, the error estimation for LES application only allows for single grid estimators for practical reasons.

Geurts and Frohlich proposed the activity parameter [10] for LES quality assessment based on the estimation of turbulent and viscous dissipation. Celik et al. [11], [12] introduced a family of Index Quality for LES error estimation, based on the effective Kolmogorov scale $\eta_{\text {eff }}$ or the eddy viscosity $\nu_{\text {eff }}$ which incorporates the contribution from the SGS model and the numerical dissipation. The estimator was extended using the proportion of resolved to total TKE based on Pope's suggestion that a "good" LES approach should resolve at least $80 \%$ of the total TKE [13]. Instead of using the proportion of resolved TKE, Antepara [14] used the residual velocity magnitude (without scaling) as an error indicator. A similar small energy density indicator depending on the residual velocity magnitude which includes the directional information was developed and employed in [15], [16]. In each of the above formulas, there is at least one parameter which requires an estimation based on empirical equations. Some error estimators were proposed based on the Richardson extrapolation approach [17], Klein assumes that in LES using implicit filtering, the modeling and numerical errors could be combined to a unique function of grid size as an alternative to assess the Index Quality. The modeling and numerical errors, and even the coupling error from the two sources, could also be separated with the help of solving the flow with a systematic grid and model variation [6], [8], which leads to a tremendous increase in the computational cost.

The current work focuses on the error estimation for practical LES mesh adaptation, thus the focus is on a posteriori single-grid error estimators which could be implemented in the post-processing process without modifying the flow solver in itself.

\section{OCTREe Flow SOLVER}

SYN3D is a finite-volume based multi-block structured flow solver for compressible flows developed at the Computational Aerodynamics Group at McGill University. The WALE Subgrid-Scale model [18] is employed for LES simulations. Modifications on the data structure and the integration process are made to carry out mesh adaptation for LES. 


\section{A. Data Structure}

To achieve mesh adaptation, we have incorporated a cellbased octree data structure [19] within SYN3D. When a certain cell is refined, eight children cells are generated with one level higher than the parent cell and a continuous sequence of memory is allocated. Given a list of refined cells from the error estimator, the connection pointers are automatically generated and the CPUs are rebalanced for parallel efficiency. In the current flow solver only geometry variables exist on face and vertices while all the flow variables are cell-based.

\section{B. Integration Process}

The time integration follows the process proposed in [19], where the time steps in refined cells are automatically reduced from that of the parent cell,

$$
\Delta t(l)=2^{l_{\min }-l} \Delta t,
$$

where $\Delta t$ is the time step on the root cell and $l$ the level of the current cell. Assuming the flux evaluation and time advancement procedures at level $l$ to be $A(l)$, the operation at level $l$ is

$$
O(l)= \begin{cases}O(l+1) O(l+1) A(l) ; & \text { if } l<l_{\text {max }} \\ A(l) ; & \text { if } l=l_{\text {max }}\end{cases}
$$

The recursion is carried on until reaching cells on the highest level $l=l_{\max }$.

\section{ERROR ESTIMATION}

In this subsection the formulas and implementation of several error estimators are presented. The approaches for estimation of numerical TKE are provided.

\section{A. Index Quality $I Q_{\eta}$}

The Index Quality based on the effective Kolmogorov scale is defined as,

$$
I Q_{\eta}=\frac{1}{1+\alpha_{\eta}\left(\frac{h}{\eta_{e f f}}\right)^{m}},
$$

where the effective Kolmogorov length scale $\eta_{\text {eff }}$ is defined by

$$
\eta_{e f f}=\left(\frac{\nu^{3}}{\epsilon}\right)^{1 / 4},
$$

where the dissipation $\epsilon$ is estimated from

$$
s=\left(\frac{\epsilon}{\nu_{\text {eff }}}\right)^{1 / 2},
$$

where $s=\sqrt{2 s_{i j} s_{i j}}$ is the square root of the double inner product of the mean strain rate tensor $s_{i j}$. The grid cell length scale $h$ is simply

$$
h=V^{1 / 3},
$$

where $V$ is the volume of the cell. $\alpha_{\eta}$ and $m$ are empirical constants.

\section{B. Index Quality $I Q_{\nu}$}

The $I Q_{\nu}$ takes the same form as $I Q_{\eta}$ does,

$$
I Q_{\nu}=\frac{1}{1+\alpha_{\nu}\left(\frac{\nu_{e f f}}{\nu}\right)^{n}},
$$

based on the eddy viscosity $\nu_{\text {eff }}$ which incorporate the contribution from the SGS model and the numerical dissipation. The implementation of $I Q_{\nu}$ can be directly fulfilled by using

$$
\nu_{\text {eff }}=\nu_{n u m}+\nu_{\text {sgs }}
$$

where the numerical viscosity $\nu_{\text {num }}$ is obtained from the numerical TKE with

$$
\nu_{\text {num }}=\operatorname{sgn}\left(k_{\text {num }}\right) C_{\nu} \Delta \sqrt{a b s\left(k_{\text {num }}\right)},
$$

with $\Delta$ the filter size and $C_{\nu}$ a constant. The estimation of $k_{\text {num }}$ will be discussed in Sec III-D.

\section{Index Quality $I Q_{k}$.}

The index quality was extended using the proportion of resolved to total TKE,

$$
I Q_{k}=\frac{k_{r e s}}{k_{t o t}},
$$

where the resolved TKE, $k_{\text {res }}$, is obtained by adding the contribution from the diagonal terms in the Reynolds stress tensor, obtained by subtracting the instantaneous flow field from the time-averaged solution,

$$
k_{r e s}=\frac{1}{2} \sum_{i=1}^{3} u_{i}^{\prime} u_{i}^{\prime}
$$

with

$$
u_{i}^{\prime}=u_{i}-U_{i},
$$

where $u_{i}$ and $U_{i}$ are the instantaneous and time-averaged velocities. $k_{t o t}$ is the sum of the resolve, modeled and numerical TKE,

$$
k_{t o t}=k_{r e s}+k_{s g s}+k_{\text {num }},
$$

where the modeled TKE, $k_{\text {sgs }}$, could be estimated from the eddy viscosity

$$
k_{s g s}=\frac{\nu_{s g s}^{2}}{C_{\nu}^{2} \Delta^{2}} .
$$

\section{Evaluation of $k_{n u m}$}

All of the above formulas depend on an estimation of numerical TKE, $k_{n u m}$ and its estimation is not straight forward. Three approaches are evaluated to achieve the goal including an empirical formula which leads to $I Q_{k-e m p}$, an approach based on the evaluation of kinetic energy dissipation which leads to $I Q_{k-k e}$ and the evaluation of turbulent kinetic energy dissipation which leads to $I Q_{k-t k e}$. 
1) Evaluation based on an empirical formula: Considering that $k_{\text {sgs }}$ and $k_{\text {num }}$ are related to the length scale $\Delta$ and $h$ respectively, Celik et al. [12] proposed a direct way of evaluating $k_{\text {num }}$ on a single grid by assuming a linear relationship between $k_{n u m}$ and $k_{\text {sgs }}$

$$
k_{n u m}=C_{n}\left(\frac{h}{\Delta}\right)^{2} k_{\text {sgs }} .
$$

In the case of implicit filtering, the filter size is directly related to the grid size, such that the only parameter to tune is $C_{n}$. The procedure is proposed based on a spectral analysis for the Decaying Isotropic Turbulence (DIT) case [20]. A process was proposed [21] to estimate the impact of the inclusion of numerical dissipation and the SGS model to the amount of captured TKE and $C_{n}$ is estimated to be 2.71 .

Although the uniform turbulence may show a linear relation between $k_{s g s}$ and $k_{\text {num }}$, the linear assumption has two primary drawbacks because of the nature of the error even if the coefficient is tuned:

- The SGS model primarily contributes to the modeled TKE in the high wavenumber region while the numerical dissipation contributes to the numerical TKE in a much wider wavenumber range;

- When approaching to the wall, the modeled TKE tends to zero, while $k_{n u m}$ should not diminish, such that $k_{s g s}$ cannot serve as a direct way to estimate $k_{n u m}$ in the near-wall region.

2) Evaluation from the kinetic energy dissipation: An improved approach was proposed to estimate $k_{n u m}$ from the kinetic energy (KE) dissipation. A practical numerical approach was proposed by Domaradzki et al. [22] and was developed in spectral space. The approach was extended in physical space by Schranner et al. [23] and the formular was proposed for explicit LES by Cadieux et al. [24]. The transport equation for the kinetic energy $e_{k i n}$ is

$$
\frac{\partial e_{k i n}}{\partial t}+\frac{\partial\left(u_{i} e_{k i n}\right)}{\partial x_{i}}=-\frac{u_{i}}{\rho} \frac{\partial p}{\partial x_{i}}+\left(\nu+\nu_{t}\right) u_{j} \frac{\partial \tau_{i j}}{\partial x_{i}},
$$

where $\nu_{t}$ is the subgrid-scale eddy viscosity, and

$$
\tau_{i j}=\frac{\partial u_{i}}{\partial x_{j}}+\frac{\partial u_{j}}{\partial x_{i}}-\frac{2}{3} \frac{\partial u_{k}}{x_{k}} .
$$

When the discretization provided by a Navier-Stokes solver is employed to estimate $e_{k i n}$, Eq 16 will result into a residual, $\epsilon_{n}$,

$$
\left(\frac{\partial e_{k i n}}{\partial t}\right)_{d}+(N S)_{d} e_{k i n}=\underbrace{\left(\frac{\partial e_{k i n}}{\partial t}\right)+(N S) e_{k i n}}_{=0}+\epsilon_{n},
$$

where $\epsilon_{n}$ is replaced by the discretization of each term in the flow solver,

$$
\begin{aligned}
-\epsilon_{n} & =\left(\frac{\partial e_{k i n}}{\partial t}\right)_{d}+(N S)_{d} e_{k i n} \\
& =\left[\frac{\partial e_{k i n}}{\partial t}+\frac{\partial\left(u_{j} e_{k i n}\right)}{\partial x_{j}}+\frac{u_{i}}{\rho} \frac{\partial p}{\partial x_{i}}-\left(\nu+\nu_{t}\right) u_{i} \frac{\partial \tau_{i j}}{\partial x_{j}}\right]_{d} .
\end{aligned}
$$

The residual $\epsilon_{n}$ serves as an estimate of the numerical dissipation of kinetic energy, since it includes the truncation error of the numerical scheme, that includes both dissipative and dispersive errors, for all terms in the kinetic energy equation. For the purpose of implementation, Eq. 19 is rewritten for the finite volume framework:

$$
-\epsilon_{n}=e_{k i n, t}+F_{e_{k i n}}+F_{a c}-F_{\nu}-\Pi+\epsilon_{\nu},
$$

where the right hand side includes the total time-rate of change of kinetic energy:

- $e_{k i n, t}=\iiint_{A} \frac{\partial e_{k i n}}{\partial t}$,

three flux terms:

- $F_{e_{k i n}}=\iint_{A} e_{k i n} u_{j} d A$,

- $F_{a c}=\iint_{A} \frac{p}{\rho} u_{i} d A$,

- $F_{\nu}=\iint_{A}\left(\nu+\nu_{t}\right) u_{i} \tau_{i j} d A$,

and two volume terms:

- $\Pi=\iiint_{V} \frac{p}{\rho} \frac{\partial u_{i}}{\partial x_{i}} d V$,

- $\epsilon_{\nu}=\iiint_{V}\left(\nu+\nu_{t}\right) \tau_{i j} \frac{\partial u_{i}}{\partial x_{i}} d V$.

In order to achieve a local cell-based error estimation, Eq. 20 is evaluated locally in each computational cell, providing local $\epsilon_{n}$ values. A numerical eddy viscosity $\nu_{\text {num }}$ is estimated by analogy to the formula for the viscous dissipation term,

$$
\epsilon_{n}=\iiint_{V} \nu_{n u m} \tau_{i j} \frac{\partial u_{i}}{\partial x_{i}} d V
$$

such that

$$
\frac{\epsilon_{n}}{\epsilon_{\nu}}=\frac{\nu_{n u m}}{\nu+\nu_{t}} .
$$

Here, $\nu_{\text {num }}$ is calculated locally and the numerical TKE, $k_{\text {num }}$, is estimated from $\nu_{\text {num }}$ with the help of Eq. 9; while, $\epsilon_{\nu}$ and $\epsilon_{n}$ are computed at each time step in a LES simulation. The time and space averaged values of $\epsilon_{\nu}$ and $\epsilon_{n}$ are then used to estimate a time and space averaged numerical TKE.

The process of $\epsilon_{n}$ evaluation is similar to the residual evaluation procedure in the flow solver. The implementation of the approach could easily be carried out for an arbitrary flow solver. However, the approach shows two drawbacks when applied for mesh adaptation for LES:

- Due to the nature of the term, the dissipation $\epsilon_{\nu}$ is always positive and $\Pi$ is zero for incompressible flow; the unsteady term $e_{k i n, t}$ tends to zero after time averaging for a statistically steady case, while the flux terms, $F_{e_{k i n}}, F_{a c}$ and $F_{\nu}$, have the possibility of showing negative values, such that the whole numerical dissipation could result in negative values in certain regions where the dissipative effect is negligible compared to the convective effect. For example, $F_{e_{k i n}}$ and $F_{a c}$ could become the leading terms in the laminar region with high mean flow velocity and low turbulent intensity as well as low velocity gradients, and lead to negative numerical dissipation [25];

- The current approach is focused on kinetic energy dissipation which incorporates both mean flow kinetic energy dissipation and turbulent kinetic energy dissipation, and both parts contribute to the evaluation of $\nu_{\text {num }}$. However, the error estimator $I Q_{k}$ should be only formulated based on numerical dissipation of the turbulent kinetic energy. 


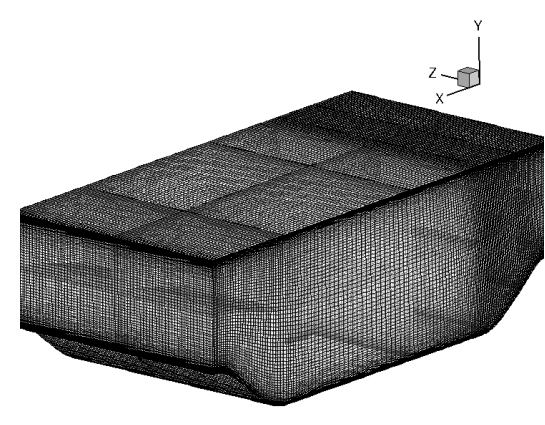

Fig. 1: Periodic hill case geometry.

3) Evaluation from the turbulent kinetic energy dissipation: In order to provide for an improved approach for an appropriate estimation of $k_{n u m}$, we propose to study the discretized resolved turbulent kinetic energy equation for LES. When using an eddy viscosity model with the inclusion of $\nu_{t}$, the time averaged resolved TKE equation for LES is written as:

$$
\begin{aligned}
\frac{\partial k}{\partial t} & +\frac{\partial}{\partial x_{j}}\left(k u_{j}+\frac{u_{j}^{\prime} p}{\rho}\right)=-u_{i}^{\prime} u_{j}^{\prime} \frac{\partial \overline{u_{i}}}{\partial x_{j}}+\left(\nu+\overline{\nu_{t}}\right) \frac{\partial^{2} k}{\partial x_{j} \partial x_{j}} \\
& -\left(\nu+\overline{\nu_{t}}\right) \frac{\partial u_{i}^{\prime}}{\partial x_{j}} \frac{\partial u_{i}^{\prime}}{\partial x_{j}}+\nu_{t}^{\prime} u_{i}^{\prime} \frac{\partial^{2} \overline{u_{i}}}{\partial x_{j} \partial x_{j}} \\
& +1^{s t} \text { order fluctuation terms, }
\end{aligned}
$$

where $\bar{u}_{j}$ means time averaged value, $u_{j}^{\prime}$ means fluctuation value and $u_{j}$ means the instantaneous value. All the $1^{\text {st }}$ order fluctuation terms disappear when the time averaging is carried out. Using the same technique as the previous subsection, we express the numerical dissipation of TKE as the discretization of all the terms of Eq. 23 in the flow solver,

$$
\begin{aligned}
-\epsilon_{n} & =\left(\frac{\partial k}{\partial t}\right)_{d}+(N S)_{d} k \\
& =\left[-u_{i}^{\prime} u_{j}^{\prime} \frac{\partial \bar{u}_{i}}{\partial x_{j}}+\left(\nu+\overline{\nu_{t}}\right) \frac{\partial^{2} k}{\partial x_{j} \partial x_{j}}\right. \\
& \left.-\left(\nu+\overline{\nu_{t}}\right) \frac{\partial u_{i}^{\prime}}{\partial x_{j}} \frac{\partial u_{i}^{\prime}}{\partial x_{j}}+\nu_{t}^{\prime} u_{i}^{\prime} \frac{\partial^{2} \bar{u}_{i}}{\partial x_{j} \partial x_{j}}\right]_{d},
\end{aligned}
$$

which is re-written for implementation purpose,

$$
-\epsilon_{n}=k_{t}+F_{k}+F_{a c}-F_{\nu}-F_{\text {inter }}-\Pi+P+\epsilon_{\nu}+\epsilon_{\text {inter }},
$$

where the additional terms compared to Eq. 19 are:

- $P=\iiint_{V} \frac{p^{\prime}}{\rho} \frac{\partial u_{i}^{\prime}}{\partial x_{i}} d V$,

- $F_{\text {inter }}=\iint_{A}\left(\nu+\nu_{t}\right) \bar{u}_{i} \tau_{i j} d A$,

- $\epsilon_{\text {inter }}=\iiint_{V}\left(\nu+\nu_{t}\right) \tau_{i j} \frac{\partial \bar{u}_{i}}{\partial x_{i}} d V$.

Dissipated TKE due to the existance of numerical error is estimated using a local length scale $l_{\text {scale }}$ and velocity scale $u_{\text {scale }}$,

$$
k_{\text {num }}=\epsilon_{n} \frac{l_{\text {scale }}}{u_{\text {scale }}} .
$$

Considering that the numerical dissipation happens at the cell size scale and the velocity fluctuation scale, we employ

$$
l_{\text {scale }}=V^{\frac{1}{3}}
$$

$$
u_{\text {scale }}=\left|\overline{u^{\prime}}\right|=\sqrt{\frac{2}{3} k_{\text {res }}},
$$

where $V$ is the local cell volume and $k_{\text {res }}$ is the local resolved TKE.

\section{NUMERICAL SET-UP}

Our primary test case is the periodic hill, with the geometry and mesh shown in figure 1, with periodic boundary conditions in both streamwise and spanwise directions, and no-slip conditions are applied at the upper and lower boundaries. The Reynolds number based on the hill height and mean bulk velocity at the hill crest is $R e=10600$. A pressure forcing term [21] is added to the streamwise momentum and energy equations in order to drive the flow to maintain a constant Reynolds number during the simulation. The flow is highly unsteady featuring separation from the continuous surface and the separation point oscillates over a large range on the wall. The mean flow is characterized by a separation bubble with an established separation and reattachment point. The form of the bubble depends on two factors: the position of the separation point and the turbulent intensity on the top of the bubble, which determines the level of energy exchange from the mean flow to the bubble region. The experimental data [26], a LES with wall function on fine grid of 4.6M [27] and a very fine wall-resolved LES on 13.1M grid [28] are available as reference data.

Two levels of grids are available for the study. The coarse mesh (MESH1) has $160 \times 160 \times 64$ grid points, while the fine mesh (MESH2) is refined from MESH1 only in the spanwise direction. The first layer of cells satisfies $y^{+} \approx 1$, which ensures the wall-resolved LES on both the upper and the lower surfaces. The goal of the study is to apply the error estimators to the coarse mesh based on the averaged solution and refine only $5 \%$ of cells with the largest error.
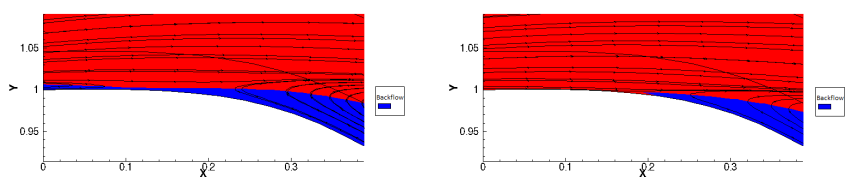

Fig. 2: Backflow on the hill top region, Left: coarse mesh; right: fine mesh.

\section{Results}

The simulations were launched on coarse and fine grids for stabilization over 100 flow through periods, and the flow field is averaged over 30 flow through periods in time and the spanwise direction. Fig. 7 (a) shows the size of the separation bubble with the contour of the captured TKE. The coarse mesh shows an early flow reattachment due to an over-estimation of the TKE level in the region above the separation bubble, the phenomenon is also reported in [27] for a coarse grid. A refinement in the spanwise direction allows for a lower TKE level and a better capture of the bubble length as shown in 


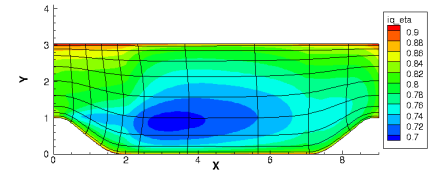

(a)

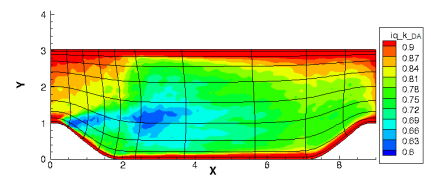

(c)

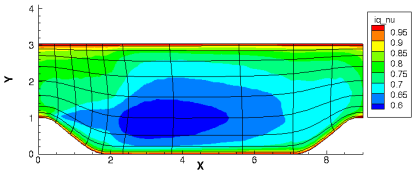

(b)

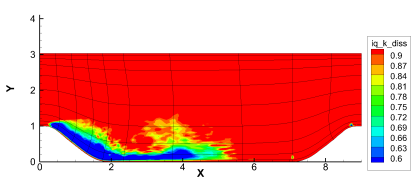

(d)

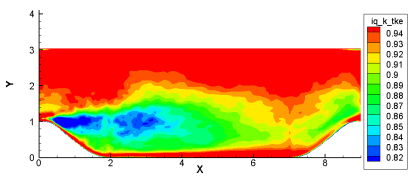

(e)

Fig. 3: Error estimation on coarse mesh based on (a): $I Q_{\eta}$; (b): $I Q_{\nu} ;$ (c): $I Q_{k-e m p}$; (d): $I Q_{k-k e}$; (e): $I Q_{k-t k e}$.

TABLE I: Separation and reattachment points for the periodic hill case.

\begin{tabular}{cllll}
\hline Mesh & Size & $(x / h)_{\text {sep }}$ & $(x / h)_{\text {reat }}$ & $L_{\text {bubble }}$ \\
\hline LES [27] & $4.6 \mathrm{M}$ & 0.22 & 4.72 & 4.5 \\
LES [26] & $13.1 \mathrm{M}$ & 0.19 & 4.69 & 4.5 \\
Coarse & $1.6 \mathrm{M}$ & -0.93 & 4.06 & 4.99 \\
Fine & $3.3 \mathrm{M}$ & 0.22 & 4.67 & 4.45 \\
$I Q_{\eta}$ adapted & $2.1 \mathrm{M}$ & 0.14 & 3.95 & 3.81 \\
$I Q_{k-e m p}$ adapted & $2.1 \mathrm{M}$ & 0.21 & 4.41 & 4.2 \\
$I Q_{k-k e}$ adapted & $2.1 \mathrm{M}$ & -0.93 & 4.99 & 5.92 \\
$I Q_{k-t k e}$ adapted & $2.1 \mathrm{M}$ & 0.22 & 4.55 & 4.35 \\
\hline
\end{tabular}

Fig. 7 (b). As is shown in Fig. 2, the coarse mesh wrongly captures a backflow layer on the top of the hill while the error is avoided by the spanwise refinement in the fine mesh. Table. I also confirms that the coarse mesh wrongly predicts the separation point by estimating the separation prior to the top of the hill at a negative $X$ coordinate and predicts an early reattachment of the flow, while the fine mesh shows good agreement with the reference LES data, even with a comparatively smaller grid size.

\section{A. Evaluation of numerical TKE}

The numerical dissipation is evaluated using all three approaches presented in Section. III-D and the results are shown in Fig. 4. It can be observed that all approaches show the same scale of numerical dissipation. The empirical formula only highlights the mixing layer above the separation bubble. The KE dissipation based approach highlights the intensively turbulent region near the separation point shows, while not targeting the mixing layer between $2<X<3.2$. The approach also shows high dissipation in a thin layer near the wall within the separation bubble region with $2<X<4$ and in a small near-wall region on the left side of the hill top region, where flow separation starts to appear. Generally, the region between the hills with $Y<1$ shows positive numerical
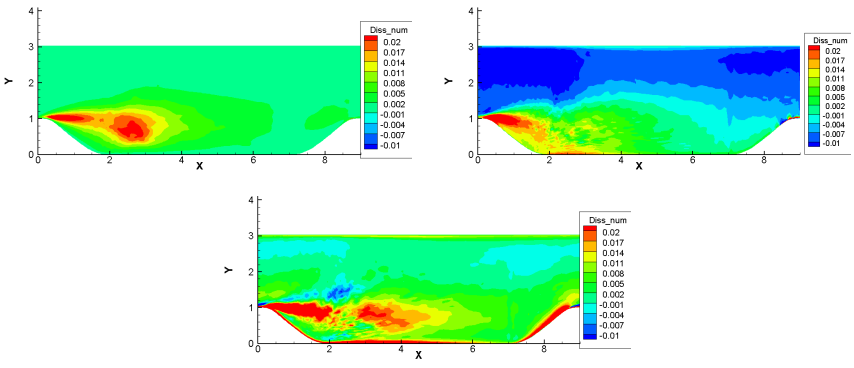

Fig. 4: Numerical dissipation, $\epsilon_{n}$, based on empirical formula (top left), KE dissipation (top right) and TKE dissipation(top right).

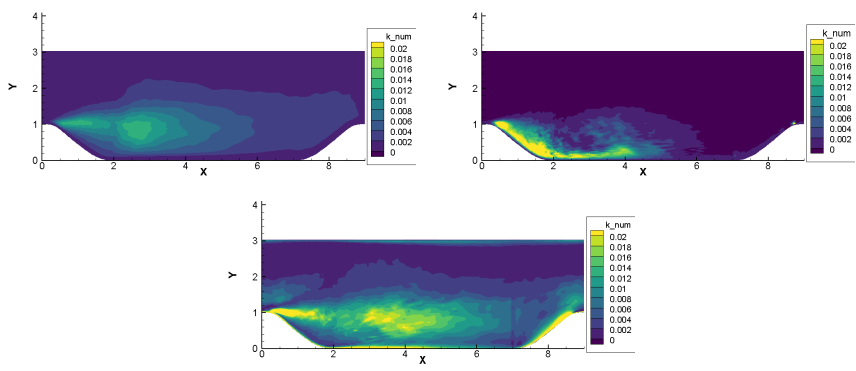

Fig. 5: Numerical TKE, $k_{\text {num }}$, based on empirical formula (top left), KE dissipation (top right) and TKE dissipation(top right).

dissipation. Negative dissipation is shown in the region above the hill for $Y>1$, where the convective flux dominates and the turbulence intensity is low, as discussed in Section III-D2. A similar trend is reported by Castiglioni and Domaradzki [25] who targeted negative numerical dissipation in the laminar region far from the wall with the current method. Referring to Eq. 20, in this region, the convective and pressure flux terms dominate over the turbulence dissipation. The TKE dissipation based approach shows high value in both the mixing layer and the boundary layer on the lower wall. In addition, the approach shows positive value or weakly negative value in the entire computational domain, even in the region dominated by the convective flux term.

The numerical TKE contour was shown in Fig. 5. The empirical formula shows high value in the mixing layer above the separation bubble with a peak value of 0.014 , while the KE dissipation based approach mainly targets the nearwall region in the separation bubble with a higher peak value of 0.044 . The comparatively low numerical viscosity in the mixing layer leads to a low numerical TKE. The TKE dissipation based approach is able to target both the mixing layer and the boundary layer with a peak value of 0.013 . The targeted boundary layer mainly includes three parts, the separation point region, the reattachment point region and a small near-wall region on the left side of the hill top region with $7.5<X<8.6$. 


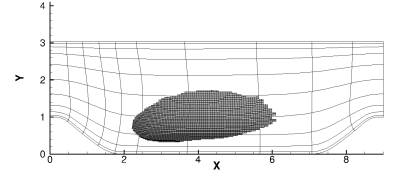

(a)

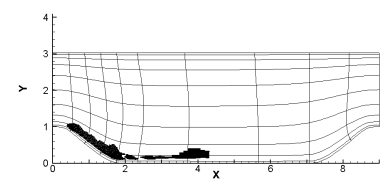

(c)

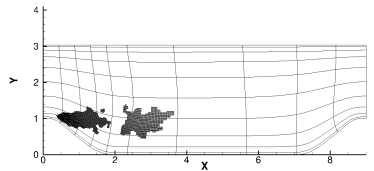

(b)

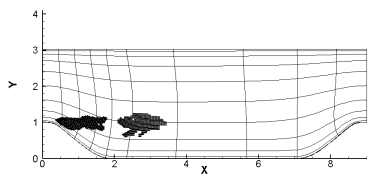

(d)
Fig. 6: (a): $I Q_{\eta}$ adapted mesh; (b): $I Q_{k-e m p}$ adapted mesh; (c): $I Q_{k-k e}$ adapted mesh; (d): $I Q_{k-t k e}$ adapted mesh.

\section{B. Error estimation}

The error estimation results based on five estimators are shown in Fig. 3. $I Q_{\nu}$ and $I Q_{\eta}$ are evaluated using the empirical formula of $k_{n u m}$. Both target a similar region in the center of the computational domain, where $\nu_{s g s}$ shows higher values and the size of the grid cells is relatively larger. A small region of mixing layer near the separation point is also slightly highlighted. The indicator $I Q_{k-e m p}$ with the empirical formula of $k_{\text {num }}$ targets primarily the mixing layer above the separation bubble. The indicator $I Q_{k-k e}$ based on the evaluation of KE dissipation provides for a different outcome, by showing high values (over 90\%) in most of the flow field and mainly targeting the near-wall region on the downstream side of the hill. Different from $I Q_{k-e m p}, I Q_{k-k e}$ does not target the mixing layer that is present above the separation bubble as a low-quality region, since the region is dominated by high strain rate and shows a low numerical eddy viscosity, $\nu_{\text {num }}$, and numerical TKE, $k_{\text {num }}$. The indicator $I Q_{k-t k e}$ based on the TKE dissipation targets both the mixing layer and the near-wall region, including the separation point and the reattachment point. Since the correct capture of TKE in those regions is essential for the correct prediction of the bubble length as shown in [27], the indicator $I Q_{k-t k e}$ is expected to aid efficiently grid adaptation for LES.

\section{Simulations on adapted grids}

Since $I Q_{\nu}$ and $I Q_{\eta}$ target similar regions for mesh adaptation while $I Q_{k-e m p}, I Q_{k-k e}$ and $I Q_{k-t k e}$ indicate different regions, we decided to carry out a comparative study based on adapted meshes based on $I Q_{\eta}, I Q_{k-e m p}, I Q_{k-k e}$ and $I Q_{k-t k e}$, which lead to four adapted meshes, namely $I Q_{\eta}$ adapted mesh (MESH3), I $Q_{k-e m p}$ adapted mesh (MESH4), $I Q_{k-k e}$ adapted mesh (MESH5) and $I Q_{k-t k e}$ adapted mesh (MESH6) shown in Fig. 6, based on the largest $5 \%$ of the error levels of the original cells. The simulation was stabilized on the adapted meshes for 40 additional flow through periods before making an average over 30 flow through periods in order to make a meaningful comparison.

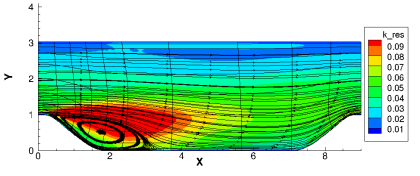

(a)

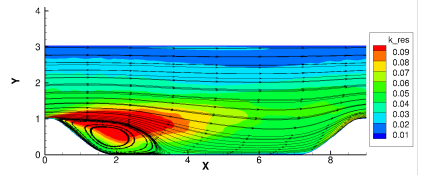

(c)

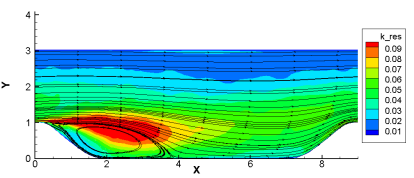

(e)

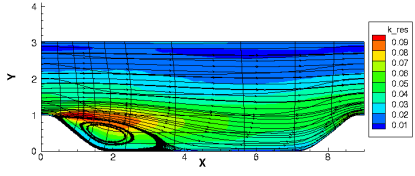

(b)

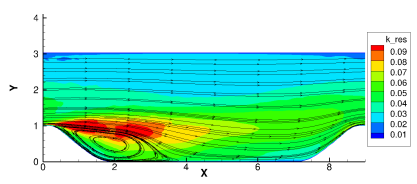

(d)

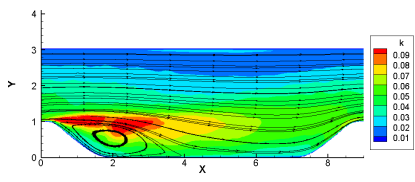

(f)
Fig. 7: Separation bubble and TKE coutour on (a): coarse mesh; (b): fine mesh; (c): $I Q_{\eta}$ adapted mesh; (d): $I Q_{k-e m p}$ adapted mesh; (e): $I Q_{k-k e}$ adapted mesh; (f): $I Q_{k-t k e}$ adapted mesh.
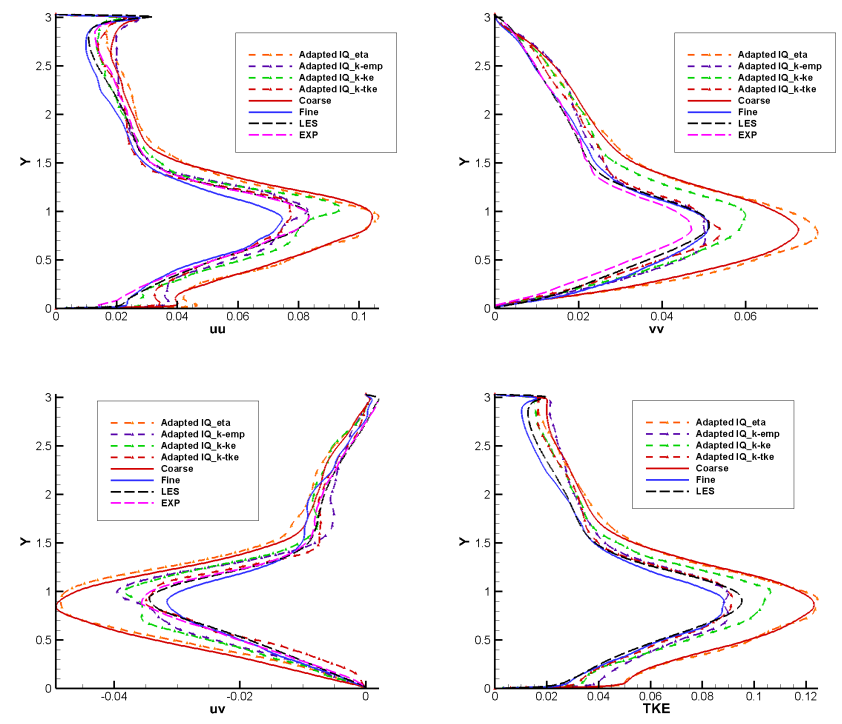

Fig. 8: Reynolds stress tensor components profiles at $X=2$.

Fig. 7 (c), (d) and (e) show the TKE field and the separation bubble captured on the $I Q_{\eta}, I Q_{k-e m p}$ and $I Q_{k-k e}$ adapted grids. It can be observed that the level of captured TKE on $I Q_{k-e m p}$ adapted mesh is significantly reduced compared to the value on the coarse grid shown in Fig. 7 (a), while the $I Q_{\eta}$ and $I Q_{k-k e}$ adapted mesh still captures a high level of TKE. Both $I Q_{\eta}$ and $I Q_{k-e m p}$ adapted meshes improve the incorrect location of the separation point, while the $I Q_{k-e m p}$ and $I Q_{k-k e}$ adapted meshes provide for a larger separation bubble, 
whose reattachment point reaches 4.41 and 4.99 respectively; while, the reattachment point captured on the $I Q_{\eta}$ adapted mesh is similar to that on the original coarse mesh as shown in Table. I. This confirms again that the size of the separation depends on the turbulent intensity captured on the top of the bubble.

Figure. 8 shows the profiles of the Reynolds stress tensor components and the TKE compared with the reference LES and the experimental data at the slice $X=2$. The studied slice spans across the main flow and the bubble regions, and is characterized by a high TKE value in the mixing layer above the bubble. The coarse mesh over-estimates all the components and wrongly captures the boundary layer while the fine mesh shows great comparison against the reference data. It is observed that the $I Q_{\eta}$ adapted mesh does not provide a discernible improvement over the coarse mesh. The $I Q_{k-e m p}$ adapted mesh shows an improvement of the captured TKE level in most of the computational domain, while still show an over-estimation of the TKE level on the lower wall region compared to the fine mesh. Due to the fact that the empirical formula estimates the $k_{n u m}$ value based on $k_{s g s}$, which tends to zero as approaching to the wall, the value of $k_{\text {num }}$ is low in the near-wall region, which leads to a reduction of $\nu_{e f f}$ and $k_{m o d}$, in the near-wall regions even if the separation and reattachment points are not targeted for refinement, which could explain the over-estimation of the TKE near the reattachment point. $I Q_{k-k e}$ targets the high value of numerical TKE near the wall in the bubble region and leads to a refinement near the reattachment point, providing for an improvement in the captured TKE level near the wall, while showing an over-estimation of TKE in the mixing layer on the top region of the separation bubble, which the estimator does not target for grid refinement. The $I Q_{k-k e}$ adapted mesh outperforms the other estimators by targeting all the essential regions including the mixing layer and the boundary layer, and by capturing the correct length of the separation bubble as well as a better level of TKE profile in the whole computational domain.

\section{CONCLUSiON AND FUtURE WORK}

In this work, several error estimators for LES have been applied to the periodic hill case in order to analyze their applicabilities and performances. The use of these error estimators is of great importance in the assessment of the quality of LES results and in the grid adaptation for LES, where the grid resolution impacts both the SGS model and the numerical dissipation, which leads to the modeled and numerical TKE. Three approaches of evaluating the numerical TKE are analyzed and implemented.

One complete grid adaptation cycle is performed for four error estimators $I Q_{\eta}, I Q_{k-e m p}, I Q_{k-k e}$ and $I Q_{k-t k e} . I Q_{\eta}$, $I Q_{k-e m p}$ and $I Q_{k-t k e}$ adapted meshes help capture the correct location of the separation point. $I Q_{\eta}$ adapted mesh fails to provide for improvement in better capture of the size of the separation bubble and the TKE level in the flow field. The $I Q_{k-e m p}$ adapted mesh successfully captures a lower level of TKE in the bubble region and a better size of the separation bubble, while provides for an over-estimation of TKE level in the near-wall region. The $I Q_{k-k e}$ adapted mesh shows better performance in the near-wall region by avoiding the over-estimation of TKE, while the estimator over-estimates the level of TKE above the bubble region. The $I Q_{k-t k e}$ is able to balance the refined region between the mixing layer above the bubble and the boundary layer, which leads to the best performance in terms of capturing correctly the Reynolds stress tensor component values and the length of the separation bubble. The future step of the study is to use residual-based and adjoint-based error estimators and to carry out simulations on adapted grids based on the combined information provided by different error estimators to provide for a better evaluation of their impacts on grid refinement.

\section{REFERENCES}

[1] B. Vermeire, "Adaptive implicit-explicit time integration and highorder unstructured methods for implicit large eddy simulation," Ph.D. dissertation, McGill University, Montreal, QC, 2014.

[2] S. Ghosal, "An analysis of numerical errors in large-eddy simulations of turbulence," Journal of Computational Physics, vol. 125, no. 1, pp. 187 - 206, 1996. [Online]. Available: http://www.sciencedirect.com/science/article/pii/S0021999196900881

[3] A. Hauser and G. Wittum, "Adaptive large eddy simulation," Computing and Visualization in Science, vol. 17, no. 6, pp. 295-304, 2015.

[4] V. John and W. J. Layton, "Analysis of numerical errors in large eddy simulation," SIAM Journal on Numerical Analysis, vol. 40, no. 3, pp. 995-1020, 2003. [Online]. Available: http://www.jstor.org/stable/4100912

[5] M. Klein, "An attempt to assess the quality of large eddy simulations in the context of implicit filtering," Flow, Turbulence and Combustion, vol. 75, no. 1-4, pp. 131-147, 2005.

[6] M. Freitag and M. Klein, "An improved method to assess the quality of large eddy simulations in the context of implicit filtering," Journal of Turbulence, no. 7, p. N40, 2006.

[7] M. Klein, J. Meyers, and B. J. Geurts, "Assessment of LES quality measures using the error landscape approach," in Quality and Reliability of Large-Eddy Simulations. Springer, 2008, pp. 131-142.

[8] T. Xing, "A general framework for verification and validation of large eddy simulations," Journal of Hydrodynamics, vol. 27, no. 2, pp. 163 175, 2015.

[9] F. Ries, K. Nishad, L. Dressler, J. Janicka, and A. Sadiki, "Evaluating large eddy simulation results based on error analysis," Theoretical and Computational Fluid Dynamics, pp. 1-20, 2018.

[10] B. J. Geurts and J. Fröhlich, "A framework for predicting accuracy limitations in large-eddy simulation," Physics of fluids, vol. 14, no. 6 , pp. L41-L44, 2002.

[11] I. Celik, Z. Cehreli, and I. Yavuz, "Index of resolution quality for large eddy simulations," Journal of fluids engineering, vol. 127, no. 5, pp. 949-958, 2005.

[12] I. Celik, M. Klein, and J. Janicka, "Assessment measures for engineering LES applications," Journal of fluids engineering, vol. 131, no. 3, p. 031102, 2009

[13] S. B. Pope, "Turbulent flows," 2001.

[14] O. Antepara, O. Lehmkuhl, R. Borrell, J. Chiva, and A. Oliva, "Parallel adaptive mesh refinement for large-eddy simulations of turbulent flows," Computers \& Fluids, vol. 110, pp. 48-61, 2015.

[15] S. Toosi and J. Larsson, "Anisotropic grid-adaptation in large eddy simulations," Computers \& Fluids, vol. 156, pp. 146-161, 2017.

[16] _ - "Grid-adaptation and convergence-verification in large eddy simulation: a robust and systematic approach," in 2018 Fluid Dynamics Conference, 2018, p. 3406.

[17] I. Celik and O. Karatekin, "Numerical experiments on application of richardson extrapolation with nonuniform grids," Journal of fluids engineering, vol. 119, no. 3, pp. 584-590, 1997.

[18] F. Nicoud and F. Ducros, "Subgrid-scale stress modelling based on the square of the velocity gradient tensor," Flow, turbulence and Combustion, vol. 62, no. 3, pp. 183-200, 1999. 
[19] A. M. Khokhlov, "Fully threaded tree algorithms for adaptive refinement fluid dynamics simulations," Journal of Computational Physics, vol. 143, no. 2, pp. 519-543, 1998.

[20] W. Rozema, H. J. Bae, P. Moin, and R. Verstappen, "Minimumdissipation models for large-eddy simulation," Physics of Fluids, vol. 27, no. 8, p. 085107, 2015.

[21] Y. Jiang and S. Nadarajah, "Assessment of error estimators for grid adaptation for LES application," in Proceedings of The Joint Canadian Society for Mechanical Engineering and CFD Society of Canada International Congress 2019. CSME-CFDSC, 2019, pp. 157-166.

[22] J. A. Domaradzki, Z. Xiao, and P. K. Smolarkiewicz, "Effective eddy viscosities in implicit large eddy simulations of turbulent flows," Physics of Fluids, vol. 15, no. 12, pp. 3890-3893, 2003.

[23] F. S. Schranner, J. A. Domaradzki, S. Hickel, and N. A. Adams, "Assessing the numerical dissipation rate and viscosity in numerical simulations of fluid flows," Computers \& Fluids, vol. 114, pp. 84-97, 2015.

[24] F. Cadieux, G. Sun, and J. A. Domaradzki, "Effects of numerical dissipation on the interpretation of simulation results in computational fluid dynamics," Computers \& Fluids, vol. 154, pp. 256-272, 2017.

[25] G. Castiglioni and J. Domaradzki, "A numerical dissipation rate and viscosity in flow simulations with realistic geometry using low-order compressible navier-stokes solvers," Computers \& Fluids, vol. 119, pp. 37-46, 2015.

[26] M. Breuer, N. Peller, C. Rapp, and M. Manhart, "Flow over periodic hills-numerical and experimental study in a wide range of Reynolds numbers," Computers \& Fluids, vol. 38, no. 2, pp. 433-457, 2009.

[27] L. Temmerman, M. A. Leschziner, C. P. Mellen, and J. Fröhlich, "Investigation of wall-function approximations and subgrid-scale models in large eddy simulation of separated flow in a channel with streamwise periodic constrictions," International Journal of Heat and Fluid Flow, vol. 24, no. 2, pp. 157-180, 2003.

[28] C. Rapp and M. Manhart, "Flow over periodic hills: An experimental study," Experiments in fluids, vol. 51, no. 1, pp. 247-269, 2011. 